\title{
12
}

\section{Realisation Issues for Brokerage}

\author{
K. Garcha \\ GPT Limited \\ New Century Park \\ Coventry \\ CV3 $1 \mathrm{HJ}$ \\ $U . K$
}

Telephone: 01203665828

Fax: $\quad 01203562638$

E-Mail: $\quad k u l @ n c p . g p t . c o . u k$

Abstract
This paper discusses three important issues related to Information Brokering:

- query processing;

- automated negotiation;

- the integration of external packages into a user's working environment.

The search, retrieval and integration of data from distributed heterogeneous databases for query processing can be very costly. This paper discusses techniques that make query processing more efficient and therefore minimise the cost. The subject of automated negotiation is discussed in terms of self interested brokers, how they can use the negotiation process to work for them (i.e. choosing the stage and level of commitment, adding conditions to decommitment, risk strategy). Technical issues that need to be considered when integrating an external application into a user's collaborative workspace are specified, i.e. communication requirements, interfaces, security, performance, dynamic operation.

\section{Keywords}

Information brokering, automated negotiation, electronic trading, query processing 


\section{INTRODUCTION}

The work that contributed to this paper, reference Garcha (1996), was conducted as a small part ( 3 man months) of the U.K. collaborative project VIRTUOSI, reference Virtuosi (1996). This project was partially funded by the U.K. Department of Trade and Industry, as part of their "LINK" programme. Partners in the project were GPT, BT, BICC, Division, Nottinghamshire County Council, GEC Hirst Research, University of Nottingham, Nottingham Trent University and Manchester University. The three-year project started in November 1993. Part of the project was aimed at supporting the fashion industry by the setting up of a commercial Brokering Service. This would provide information and services for the various interested parties in the fashion and clothing industry (e.g. fabric manufacture, accessories, finance services).

The scope of this paper is: firstly to study the way a Broker can efficiently search, retrieve and integrate data from distributed heterogeneous data sources; secondly how a Broker can successfully negotiate for information and services; and finally the technical issues that need to be considered when integrating external applications into a user's working environment.

There are other related areas of interest not covered by this paper, e.g. other Broker construction issues, Brokers and Agents relationships, Internet support for Brokerage, security of payment mechanisms.

The first section of this document gives some background information on a broker. The second section discusses query processing. The third section shows how external information or services can be negotiated. The final part of this paper considers what issues need to be resolved when integrating an external application into a user's working environment.

\section{BROKER}

This section gives some background information to the concept of a Broker, further details can be found in reference Foss (1995).

The increase in the number of commercial information services (information, software applications, entertainment, etc.) which are publicly accessible from the world's networks, has brought with it its own problems. Information service providers need to effectively market their products and services in a totally new way. A new automated trading environment is emerging, which requires new management and administration mechanisms. Users are faced with access to an 
ever increasing mountain of information but do not have the tools capable for efficiently locating, processing and managing this information.

One way of tackling these problems in an integrated manner is by using an intermediary, i.e. an information broker. A broker is not an entity that can be rigidly defined. It could be envisaged as being a freely evolving, self organising entity that would operate in a free market. Brokers would provide personalised information services to the customer by liaising between information service providers and clients. Brokers could be used as marketing entities to target the appropriate audience. The broker is in a good position to mediate the methods of interaction, apportion information rights and revenues and even administer taxes to various parties in a trading model. A broker can be used to search, negotiate, integrate, manipulate and monitor for information and services for clients, saving time and effort. One of the broker's key tasks is to interact in real-time with other brokers belonging to different organisations, buying goods and services for its clients.

It is not practical to build a single system to perform all the functions of a broker, what is required is a modular approach where co-operating specialised autonomous agents find, retrieve, integrate, analyse and present the data. The functionality of agents can vary, some agents will accept queries, and then themselves generate queries to other agents, whereas others will just answer queries (data stores). There is no clear difference between what a broker is and an agent. In our system the broker is the user's intelligent gateway to the particular information services that it provides. The broker itself could be used by other agents and brokers to provide information (i.e. a recursive relationship).

\section{QUERY PROCESSING}

The broker needs to efficiently process queries from users; retrieving and integrating distributed data can be very costly. Query processing involves developing an ordered set of operations for obtaining a requested set of data, such as selecting the information sources, choosing operations for processing the data, selecting sites where the operations will be performed and the order in which they will be performed. What is required is an automated dynamic system to generate and execute query access plans. This planner needs capabilities such as: executing operations in parallel; re-planning queries that fail while at the same time executing other queries; gathering additional information to aid the query processing and the acceptance of new queries while other queries are being executed.

Further information can be found in reference Knoblock and Ambite (1996). 


\subsection{Knowledge of a Broker}

An important aid to handling query processing is the broker's knowledge base covering its area of expertise (domain), its sources of information and details about its clients. One way of representing the knowledge of a broker is via the use of modelling techniques. These models can be used to determine how to process an information request.

\subsubsection{Domain Model}

This provides collaborating brokers/agents and any other users with an interface to the broker. The model is a description of the application domain from the point of view of users who may need to obtain information about the application domain. It contains a detailed model of its area of expertise, and the terminology for user communication. This model includes the broker's interest profile - those services that the broker is interested in finding from other brokers and agents. These services would have certain criteria associated with them: quality, credibility etc. .

\subsubsection{Source Models}

These are the resources available to a broker when information requests cannot be answered internally.

A broker will have models of other agents/brokers and information sources that provide useful information for its domain of expertise.

A source model contains three types of information. Firstly the source model contains a service profile to enable the broker to assess how the services offered by the source match its requirements. The service profile would include an affinity value, giving a measure of to what degree the services required by a broker match the services offered by the source. This value can be used by the broker to decide whether to retain the source and to prioritise the order in which sources are processed. Within the profile there would also be a general description of the services offered by the source together with information on the quality of service, reputation rating, previous customer satisfaction levels etc. Secondly there is a description of the contents of the information source (only of areas of relevance to the domain) and the language that the information source communicates with. Thirdly the relationship between the information source and the domain model is described. This relationship is used to transform domain level queries into a set of queries to the appropriate information sources. 


\subsubsection{Client Model}

The client model provides information specific to customers, e.g. interests, payment rating, satisfaction level etc.

\subsection{Steps in Query Processing}

Once a query has been received from a user (human/computer) the query can be handled as follows :

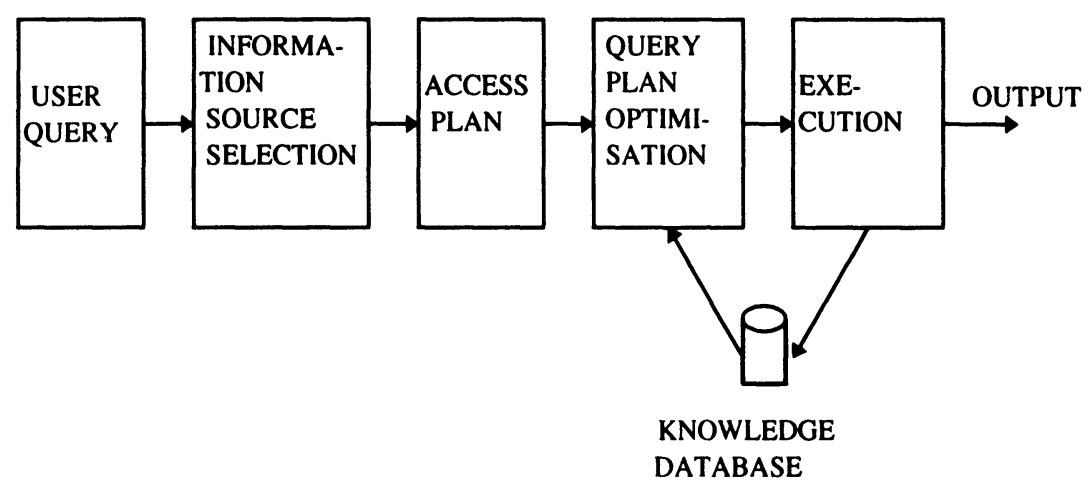

Figure 1 Query Processing.

\subsubsection{Information Source Selection}

An important and difficult problem is how to efficiently retrieve information from distributed heterogeneous multidatabase systems. Retrieving and integrating distributed data often requires processing and storage of large amounts of intermediate data, which can be costly. This cost can be reduced by using automated algorithms to reformat queries for individual databases and multidatabase systems, making them more cost effective.

The broker firstly needs to reformulate the user's query into one or more queries to specific information sources. The following reformulation operations can be used to achieve this task effectively and efficiently : 


\section{Mapping}

This maps a domain-level concept to a source-level concept. If a query directly maps onto a single source of information then the mapping is straightforward. However in some cases there may be several information sources that provide access to the same information or no single information source can provide all the required information. The broker will need to choose a solution so as to minimise the cost of the overall query.

\section{Concept Generalisation}

Concept Generalisation uses knowledge stored in the domain model, in the form of hierarchical relationships between concepts, to reformulate a query and find an appropriate data source. For example, if in the clothing industry (part of the Virtuosi project), a query requiring information about silk material is not satisfied by the silk concept, then the query could be generalised to the fabrics concept (higher up in the hierarchy), and the information obtained.

\section{Concept Specialisation}

This replaces a query specification, with a more specific query request, by checking the constraints of the query. For example if in Virtuosi (part of the project related to the fashion industry) there was a request for all suppliers who stock womens' clothes greater than size 16 , then the query could be reformulated using knowledge from the model, that only large-size-clothes suppliers have this size range.

\section{Definition Substitution}

This replaces a relation defined between concepts in the domain model with equivalent terms that are available in the source-models.

\subsubsection{Query Access Planning}

Having selected the sources of information to resolve the query, secondly the broker needs to construct a plan for retrieval of the information requested by the reformulated query by determining the appropriate data manipulation and ordering. This would involve steps such as sending a specific query to some information source, combining results from different information sources and temporarily storing partial results.

The following are some operators that can be used for data manipulation :

- Move: Moves a set of data from one broker/agent to another.

- Join: Combines two sets of data; overlapping data from ferent sources will need to be combined into one consistent data source. 
- Retrieve: Specifies the data to be retrieved from a particular information source.

- Select: Selects a subset of the data using given constraints.

- Construct: Combines existing data to form a new data item.

Data restructuring would be required, where data retrieved from one database must be transformed into the structure specified by a common schema, for integration with data from other sources. Mediation would be required to resolve semantic heterogeneity issues. An example of this would be if data on earnings from multiple sources came in monthly, weekly and hourly salaries but needed integrating into yearly earnings.

To produce a cost-effective query plan the system could use a simple estimation function to calculate the various costs of the operations.

\subsubsection{Semantic query-plan optimisation}

Knowledge gained about the contents of databases can be used to perform semantic query optimisation to minimise the execution cost of the query. The semantic knowledge can be learnt as a set of rules. Queries can be reformulated by adding, modifying or removing constraints.

E.g. Suppose a query was set-up for the clothing industry (part of Virtuosi) looking for a company who could manufacture 10,000 garments of a particular type a week. However manufacturing capacity in the data source was not indexed. Then a lot of processing would be involved in searching every record. However if the system has a rule stating that medium sized firms have the capacity to handle production of up to 12000 garments and the firm-size field is indexed, then this constraint can be used to speed up processing.

The resulting plan is more efficient and would return the same results as the original one.

\subsubsection{Execution}

At this stage the optimised query plan is executed. Queries are sent to the appropriate information sources (in parallel where possible), data is transferred, and a response is constructed for the user.

Note - the data coming back will need its metadata and be required to be and formatted so that the receiver understands the information. 


\subsubsection{Integration}

In order to get the lowest cost integration and access plan, reformulation of the queries and generation of the query access plan have to be done at the same time.

Integration of the planning and execution process can also provide many benefits :

- The broker can continuously accept and plan queries while it is executing other queries.

- If a failure occurs, an agent can re-plan the failed portion of the plan while it continues to execute queries that are already in progress. After re-planning the system can redirect the query to a different information source or agent.

- A Broker can issue actions to gather additional information for query processing, e.g. to help to select from a number of potential information sources.

\subsubsection{Learning}

A broker can improve the performance and accuracy of its query processing operation, by learning over time. Frequently used or difficult to retrieve relatively static information can be stored in cache locally, being referenced as a source directly. Brokers can learn about the contents of information sources in order to minimise the costs of retrieval. Information agents can analyse information sources regularly in order to keep their domain model up-to-date and thus provide a better service. Brokers can learn from other brokers and agents by observing their behaviour and then imitating them. Brokers can learn from activity taking place, such as changing customer interests, customer satisfaction / dissatisfaction, reliability of sources, quality of sources etc. This data can be recorded and maintained in the broker's models and used to make decisions on future activity. Brokers can use feature based (use clients' personal data) or collaborative (use data from other clients with similar interests) techniques to recommend potential useful information or services.

\section{AUTOMATED NEGOTIATION}

Negotiation is a joint decision making process in which various parties state their requirements, some of which may conflict. Negotiation allows all parties to move towards agreement by a process of concession or the search for new alternatives. 
Further information on this subject area can be found in reference Sandholm and Lesser (1995).

\subsection{Need for Automated Negotiation}

One of the broker's key tasks will be to interact in real-time with other brokers and agents belonging to different organisations, performing monetary transactions in terms of buying goods and services for its clients. The broker will also be interested in forming short term alliances with other brokers and agents in order to respond to more diverse requests from users than it individually could. Through forming these alliances the broker can take advantage of economies of scale without suffering from diseconomies of scale.

Negotiation relies heavily on the ability of agents to communicate and to understand each other. Messages need to be standardised by building common ontologies, message wrappers, etc.

\subsection{Types of Multi-Agent Negotiation}

There can be various types of multi-agent negotiation depending on the type of environment the broker is dealing with. If the broker is dealing with agents that have only the broker's interests in mind (e g. broker's internal agents) then the broker will be working in a co-operative environment, where the broker should try to minimise costs and maximise revenue of the operation as a whole by sometimes accepting local losses.

When the broker is dealing with agents in a virtual organisation, then the broker will be working in a self-interested environment concerned with maximising its own profit. The brokers and agents within this set-up will only take action for a payment and may not be totally honest whenever it benefits them. They will maintain their own local decision autonomy as they have their own private goals.

The following sections explore automated negotiation among self-interested agents that make negotiation decisions in real-time with limited resources.

\subsection{Interaction Protocol}

Automated negotiation is currently in its early stages. There is no clearly defined interaction protocol enforced by law (as in traditional negotiation) and when laws are defined there is the potential problem of agents in different countries being 
governed by different laws. Laws may not be strictly enforced (enforcing them may be impracticably expensive). A computer agent can vanish at any point in time - laws can only be enforced if the terminated agent represented some real world party and the connection between the two can be traced. To address this issue some systems tie each agent to its real world party.

A broker is in an ideal position to enforce an interaction protocol by acting as a trusted intermediary, making sure any laws are adhered to and any other business requirements such as accounts, taxes are also met.

Automated trading can be made to work more effectively by splitting up the delivery of larger goods into smaller deliverables, making it less tempting for each trading partner to defect. Discounts, lateness penalties, deadlines, etc. Can be used to motivate a trading partner to respond in a timely manner.

\subsection{Contracting Scenario}

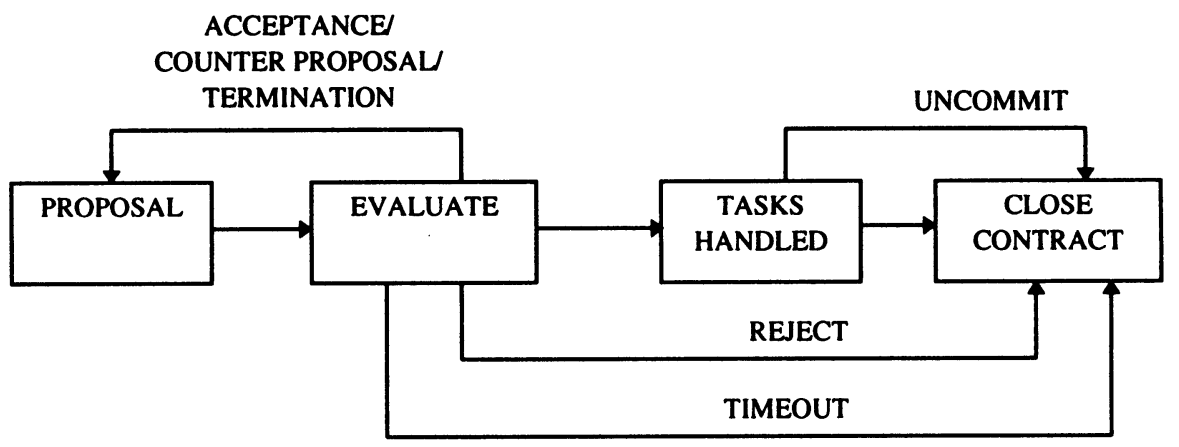

Figure 2 Negotiation Process.

Negotiation can start with either a contractor or a contractee message. For illustration purposes we will assume the contractor sends out the initial message. A contractor's message can specify alternative contracts that the contractor is willing to commit to. Having received the message the contractee can respond in one of the following ways :

A contractee can accept one of the options of the contractor's message by returning a message identifying the negotiation, option and specifying the accepted terms and conditions (tasks, delivery times, quality, payment, commitment, etc.). Once the contractee has accepted, then the contractor is automatically committed to paying the amounts specified and can cancel the deal on a task set only by 
paying the contractee a penalty. Also once the contractee has accepted an option the contractor becomes decommitted from all the other alternatives it suggested.

If the contractee decides not to accept any of the options and wants to terminate the negotiation, then a terminate message for the specified negotiation should be returned. As a result the contractor becomes decommitted from all of the options it proposed.

The contractee may decide to send a counterproposal that the contractor can either accept, further counterimpose or terminate the negotiation.

\subsection{Commitment and Negotiation}

Commitment means that one agent binds itself to a potential contract while waiting for the other agent to either accept or reject the offer. If the other party accepts then both parties are bound to the contract.

\subsubsection{Stage of Commitment}

Traditionally commitment takes place in the bidding phase. If the bidder was awarded the task then the bidder has to take care of it at the price mentioned. Computer agents can be designed so that commitment can take place at any stage of the negotiation process. The choice of commitment can be a static protocol design or it can be decided dynamically.

\subsubsection{Levels of Commitment}

The level of commitment can also be negotiated over. Commitments can be assigned a level of commitment based on monetary commitment breaking cost. This cost can be made dependent on other variables such as time, events in other negotiations, etc. This enables a broker to take risks. With a low level of commitment the broker would be able to accept a job and later try to contract out parts of the job. With full commitment the broker would have to be able to handle the job itself or have standing offers from agents/brokers that it is able to contract the task to.

Making low commitment offers to multiple agents gives the broker a greater chance of getting its offer accepted quickly while only having to pay a minimal penalty if there is more than one acceptance. 


\subsection{Conditional Response Time}

By adding a condition to the response time, the contractor can motivate the negotiation partner to respond quickly. This can be done by a strict deadline or time-dependent payment scheme. In the strict deadline method the contractor specifies a date that a particular option is available for. If the negotiation partner has not answered by that time the sender of the message gets uncommitted from that option. The time-dependent payment scheme allows a contractor to describe payments that decrease as the acceptance of a contractor message is postponed. In the case of a contractee it allows it to specify payments that increase as the acceptance of a contractee message is postponed.

A time-dependent lateness penalty schedule similar to the above car : used for delivery of goods and services to a specified time.

\subsection{Accepting/Waiting}

An agent does not know what offers it will receive in the future but a negotiating agent needs to consider the trade-off between accepting early or waiting for better offers. By waiting an agent may receive better offers later. Having more options available would enable an agent to make a more informed decision. A disadvantage of waiting is that the agent may lose the contract through some other agent accepting the offer, or may be paid less for the same job. If a response deadline is missed the negotiation terminates. The agent can begin a new negotiation on the same issues, but it will not have the other agent's commitment at first.

\subsection{Terminating the negotiation}

Negotiation can go on indefinitely. Knowing when to terminate is difficult. One way of deciding this is for an agent to stop negotiating once it has made no contracts during a certain fixed number of negotiation iterations. Some agents may use an estimating scheme to decide the feasibility (resources and cost) of handling the operation. Negotiation can be terminated by a "strict deadline" method whereby if the negotiation partner has not responded by a certain date the negotiation ends. In some cases where an agent for some reason cannot achieve the requested task and an important business partner is involved it is in the interest of the agent to send a terminate message. 
It is important to take account of overheads likely to be incurred in the negotiating process, as in some cases these may outweigh any benefits gained from the contract.

\subsection{Risk strategy}

Varying the level of commitment allows a wider variety of negotiation risk management techniques.

High levels of commitment can be used to reduce the risk of failure via adding severe penalties for breaking the contract.

Low levels of commitment allow agents to take the risk of accepting a task without fully knowing how and whether they are going to be able to achieve the task, because the cost of failure is inexpensive.

Using a level of commitment an agent can make the same offer to multiple agents, thereby increasing the chance of acceptance. If there is more than one acceptance the penalty is minimal.

An agent has to decide what level of feasibility checking it should do before accepting, if too much time is spent another agent may win the contract before the reply is sent. If too little time is spent the agent may make an un-beneficial contract.

As agents have limited resources they will need to decide which tasks to work on and in which order. They may want to put more resources on some selective more worthwhile potential contracts and ignore some of their existing obligations.

A group of agents can accomplish a big operation if they take the risk of working together. The level of risk being reduced by adding penalties for any breach of contract.

\subsection{Anticipating the future}

An intelligent agent should anticipate future negotiation events in its negotiation strategy.

An agent needs to consider during initial negotiations if the cost or feasibility of carrying out a task depends on the carrying out of other future tasks. One single contract may not be beneficial (loss maker) but further potential orders in the 
future may add up to a large profitable contract. Payments and commitment functions can be set on future events.

In some cases it may be advantageous to work out the feasibility of a task in all possible future scenarios. Where different combinations of to-be-sent and to be received offers have been accepted, different combinations of old and to occur offers have been broken and different domain events have occurred.

\subsection{Agent's Knowledge}

Agents need knowledge to deal with the negotiation process. A knowledge database can provide information on profiles of its client, information on past dealings, commercial preferences, strategy, constraints, etc., to help make decisions.

For further information reference section 3.1 Knowledge of a Broker.

\section{INTEGRATION OF EXTERNAL APPLICATIONS}

One of the main functions of a broker will be to integrate external applications into a multi-user virtual environment. This enables applications to be used interactively for remote team based activities enabling real time arbitration, communication and co-operation. Each user can edit the contents with each action being reflected to all the other users. A team of designers of a product, located in different places can meet in their work environment and interactively collaborate on the viability of different designs, in terms of cost and profitability.

Further information on this subject area can be found in references DR. Furness, Dr. Kawahata (1996) and Amselem (1996).

\subsection{Requirements}

The following are technical issues that need to be considered when integrating external applications into a multi-user Virtual Environment:

\section{Communication}

If the application is to run on the same platform as the virtual environment, does the application support that platform? If networking is involved is the required network technology supported? 


\section{Interfaces}

Are the interfaces and $\mathrm{I} / \mathrm{O}$ devices (including various types of media) required by the application supported by the virtual environment. Does the environment support open collaborative applications, i.e. the sharing of documents, images etc.

How flexible is the virtual environment in terms of its user interface? For example would it provide translation facilities for an application requiring audio but where the user has no audio facility? (The virtual environment could convert the audio to text for the user).

\section{Security}

Is the required level of security provided by the virtual environment ?, e.g. access only allowed to users specifically granted access to the application.

\section{Performance}

Are there enough resources to drive this application at a satisfactory level of performance?

\section{Distributed Environment}

Can the application run on a different machine to the virtual environment and the user without overloading the network with data being passed between users ?

\section{Network Communication Model}

Users in a distributed multi-user application sharing the same virtual space interactively require their host machines to communicate with each other using a network. Virtual environments are based on certain types of network models, e.g. Centralised network model, Distributed network model, Broadcast network model. These models define the architecture of the network, e.g. client/server or peer to peer, the way the messages are communicated and to whom. Each approach has its advantages and disadvantages. It needs to be considered how well the network communication model supported by the virtual environment suits your application. For example, if the virtual environment uses a distributed network communication model (peer to peer) it can be difficult to maintain database consistency. In this type of model each peer maintains a local copy of the database, so when changes are made to the database, the peer must communicate these changes to all other peers in the system.

\section{Number of Users}

How many users can effectively share the same Virtual Environment compared to what the application is designed for?

\section{Dynamic loading of applications}


Will the system allow dynamic loading and updating of applications as they are required?

Dynamic connection of users to the system

Does the virtual environment allow users to $\log$ in and out of the system as required while running?

\subsection{Tools}

Tools that make integration easier are emerging, which enable applications to be run on any platform.

\subsection{Administration Issues}

Having decided that a particular piece of software can be integrated, a contract needs to be produced for the licensed use of the software, upgrade payment details etc.

\subsection{Disintegration}

On expiration of the licence, a mechanism is needed to disable the software from the system and clear up any payments due.

\section{SUMMARY AND CONCLUSIONS}

It can be seen that the broker has an important role to play in the further evolution of the Global Information Infrastructure. This paper has discussed the issues of automated negotiation, integration and query processing that are seen as being crucial to the realisation of Information Brokerage.

GPT are looking at further developing this subject area by developing a prototype with Queen Mary Westfield of London (University) and also carrying out further research as part of an ACTS brokerage programme.

\section{REFERENCES}

Amselem D. A. (1996) Window on Shared Virtual Environments. 
http://www.afit.af.mil/Schools/En/ENG/LABS/GRAPHICS/annobib/writ eups/amse95-00.taa.html

Dr. Furness and Dr. Kawahata (1996) GreenSpace project. http://www.hitl.washington.edu/projects/greenspace

Foss J. (1995) Agents in Information Brokering Services, UNICOM Seminar on Business Applications of Intelligent Agent Technology, London.

Garcha K. (1996) Realisation Issues for Brokerage, Virtuosi, GPT Limited.

Knoblock C. and Ambite J. (1996) Agents for Information Gathering, http://www.isi.edu/sims/knoblock/info-agents.html

Sandholm T. and Lesser V. Issues in Automated Negotiation and Electronic Commerce.

http://dis.cs.umass.edu/research/ecommerce.html

Virtuosi Project (1996),

http://www.crg.cs.nott.ac.uk/Virtuosi

Acknowledgements - I would like to thank Jerry Foss of GPT Limited for his help.

\section{BIOGRAPHY}

Kulwinder Garcha joined GEC in 1987 during the following year GPT was formed from the amalgamation of GEC and Plessey Telecommunications. She has experience at all levels ranging from research to implementation. She has worked on various projects including, systems integration, mobile telecommunications (Telepoint and GSM) and Intelligent Networks. 